\title{
Design of a Mobile, Inexpensive Device for Upper Extremity Rehabilitation at Home
}

\author{
James S. Sulzer, Michael A. Peshkin and James L. Patton
}

\begin{abstract}
Chronic stroke survivors lack sufficient outpatient therapy, despite indications that more therapy at the chronic stage can restore some function. Both insurance and physical constraints on therapists prevent training in the home, most likely where this activity would take place. Nevertheless, this gap reveals a promising application for robots, low-cost home care. A robot designed for home use needs to be inexpensive, portable and safe. Earlier, we have explored a type of compliant variable transmission known as the MARIONET (Moment arm Adjustment for Remote Induction Of Net Effective Torque). The proof-of-concept, behaving similar to a rotary Series Elastic Actuator, has been found suitable for low-cost, light weight applications. This paper discusses further analysis of the singlejoint MARIONET and proposes the design for the new planar, upper extremity two-joint manipulandum for clinical and home use.
\end{abstract}

\section{INTRODUCTION}

For most stroke survivors, the need for rehabilitation after outpatient care doesn't go away. Outpatient therapy ends after 2-6 months. Yet research has indicated that even during the chronic stage, potential for motor recovery is not lost [1],[2],[3]. Since insurance will not pay for therapists to work with chronic patients, we can look to robots to fulfill this need. Unfortunately, few robots currently exist that are affordable and portable enough for patients to take home or even for widespread use in clinical settings. In addition to low cost and small size, a robot made for private use must be able to mitigate any safety concerns.

Initial applications of robotics have focused on positioncontrolled industrial applications, not necessarily fit for human interaction. Zinn et al. examined the combination of robot arm inertia and interface stiffness to gauge the Head Injury Criteria, the metric used to determine the star rating in automobile crash tests, at an impact speed of $1 \mathrm{~m} / \mathrm{s}$ [4]. They found that the PUMA 560, an industrial robot used in many human interactive experiments, is capable of causing severe head trauma. Using this example, a device with low inertia and low interface impedance would result in a safer robot.

Robots like the PUMA 560 have high impedance because they use gear reductions on their motors, enabling them to

This work is supported by the American Heart Association

J. Sulzer is with the Department of Mechanical Engineering, Northwestern University, Evanston, IL 60208, USA and the Rehabilitation Institute of Chicago, Chicago, IL, 60611 USA sulzer@northwestern.edu

M. Peshkin is with Faculty of Mechanical Engineering, Northwestern University, Evanston, IL 60208, USA peshkin@northwestern.edu

J. Patton is with Faculty of Mechanical Engineering and Biomedical Engineering, Northwestern University, Evanston, IL 60208, USA and the Rehabilitation Institute of Chicago and Physical Medicine and Rehabilitation, Chicago, IL 60611, USA j-patton@northwestern.edu use smaller, less expensive actuators and achieve the same torques. But the gain in torque results in higher impedance and lower speed. One could develop software to provide compliance, but this effort may be stymied by finite sampling rates, transmission dynamics and backlash. Steel cable transmissions have negligible backlash and retain lower interface impedance, but are rather expensive to produce and maintain.

An interesting solution to this problem has been in use for over a decade; the higher apparent inertia and impedance from gearmotors can be reduced by incorporating a series elastic element on the actuator. First developed by Pratt et al. [5], Series Elastic Actuators (SEAs) have been used in a variety of machines including lower extremity orthoses and bipedal robots [6],[7],[8],[9],[10]. Some of these SEAs even have control over the compliance of its elastic element [4],[11]. SEAs dampen torque ripple, backlash and other undesirable position errors, but sacrifice bandwidth and position accuracy as a result. For relatively low bandwidth applications like human movement, SEAs are an effective choice.

Besides reducing interface impedance, reducing inertia also enhances safety. The actuators themselves are one of the heaviest parts of a robot, so moving them to the base and driving the load remotely is safer. Combined with using gearmotors, the device becomes lighter and less expensive. Its light weight, low cost and safety are key in developing a home therapy system.

A solution to these criteria has been introduced earlier. The MARIONET (Moment Arm for Remote Induction Of Net Effective Torque) is a novel transmission that uses moment arm manipulation to induce torque, but can also mechanically control its compliance [13]. Performance evaluation of a single-joint proof-of-concept has shown that it is capable of accurate torque control and position control. This paper will reintroduce this novel actuator, and present the plans for the next generation, a two-joint planar robot for home use.

\section{MARIONET PROOF-OF-CONCEPT}

Torque of a cable-driven joint may be modified in either of two ways: by changing the tension in the cable, or by changing the moment arm, the cable's line of action, on the joint. The MARIONET employs both of these methods into a novel actuator that behaves similar to a rotational SEA. A canonical version of the proof-of-concept is shown in Figure 1. The device is composed of a Rotator (providing a rotational constraint) on the Pulley, a Link or Arm behaving as the end effector, and two actuators, one controlling the tension in the cable passing through the pulley, and one 


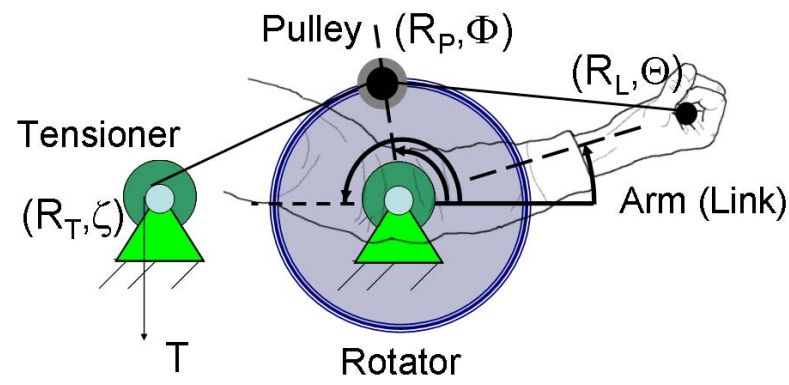

Fig. 1. Canonical illustration of the MARIONET. The MARIONET focuses on manipulating the moment arm of a cable-driven joint. The version shown here is capable of adjusting the line of action of the cable in a rotational manner, through the motor-driven Rotator. A tensioning motor provides control of compliance in the joint. Note polar coordinates used to describe positions of the Tensioner, Pulley, and Link.

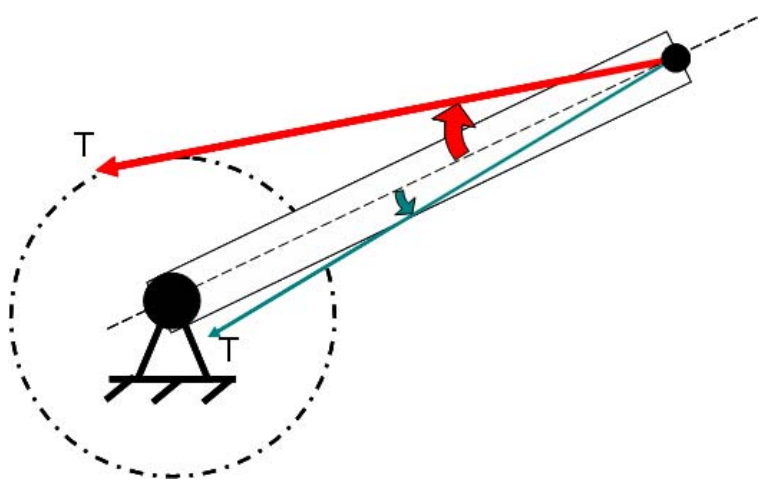

Fig. 2. Larger moment arms exert more torque. Two cases are shown in this illustration: The thick red arrow presents the case of a larger moment arm, which results in more torque, and the blue arrow exhibits the opposite case. The tension is the same in both cases. Also note that zero torque occurs when the arrow passes through the joint center.

controlling the position of the Rotator. This section will characterize how this device produces torque on its user, finding it adaptable to different subjects and capable of both error augmentation and guidance training regimens.

\section{A. Characterization of the MARIONET}

The function of the MARIONET is to exert torque on its subject; this can be broken down by examining each way torque is modified. Figure 2 illustrates using a rotational constraint on moment arm modification. The cable tension, shown as long, directional arrows, must pass through the constraint, shown as a dotted circle. Given constant tension, the cable exerts a larger torque on the end effector as the moment arm increases. The torque relationship can also be expressed mathematically in 1 :

$$
\tau=\frac{R_{L} R_{P}}{L_{L P}} \sin (\theta-\phi) T,
$$

where

$$
L_{L P}=\sqrt{R_{L}^{2}+R_{P}^{2}-2 R_{L} R_{P} \cos (\theta-\phi)},
$$

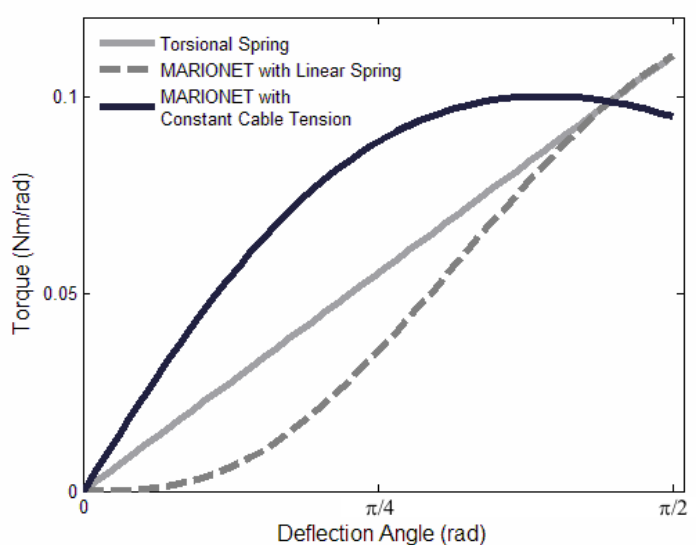

Fig. 3. Torque-deflection curve using rotational constraint compared to torsional spring.The torque-deflection curves from the rotational constraints in Figure 2 show a sinusoidal relationship, differing from the linear relationship of a torsional spring. Note that a linear spring results in a decrease of sensitivity to deflection, while constant tension results in an increase of sensitivity. Typical values for $R_{L}(0.3 \mathrm{~m}), R_{P}(0.1 \mathrm{~m})$, cable tension of $10 \mathrm{~N}$, and spring constant $(10 \mathrm{~N} / \mathrm{m})$ were used in the calculations.

from Figure $1, R_{L}$ and $R_{P}$ are the distances from center to the end effector and constraint, respectively, $\theta$ and $\phi$ are the absolute angular distances from the datum, and $T$ is the tension in the cable. In the case of an elastic cable, $T=k x$, where $k$ is the spring constant and $x$ is the displacement. The MARIONET is designed to control two of the variables in this equation: the angular distance $\phi$ and the cable tension, $T$, affecting the output, $\theta$. The difference between $\theta$ and $\phi$ will be referred to as the deflection.

The torque-deflection curve of the examples in Figures 1 and 2 differ from using a torsional spring, as shown in Figure 3. The curve of the rotational constraint combined with a linear spring behaves as a low-pass filter to any undesired deflections. This is one possible paradigm for the MARIONET. Figure 1 uses a Tensioner motor to directly control cable tension. This version is more sensitive to small deflections than the torsional spring.

As stated earlier, torque in a cable-driven joint can also be changed by modifying the tension in the cable, or the pretension in the case of an elastic cable. The amount of pretensioning in the elastic cable can offset the sensitivity of the torque-deflection curve, as seen in Figure 4. Each one of these curves can be seen as a different resistance level for training. Thus, with control over both moment arm and cable tension, the MARIONET is capable of adapting to a wide array of subjects.

A Tensioner and Link located opposite each other, as in Figure 1, can result in an singularity caused by the nonlinear constraint of the Rotator. This instability can be avoided in control, as detailed in prior work [12].

\section{B. Performance of Proof-of-Concept}

A single-joint proof-of-concept for the MARIONET has been developed and tested in previous work [13]. This 


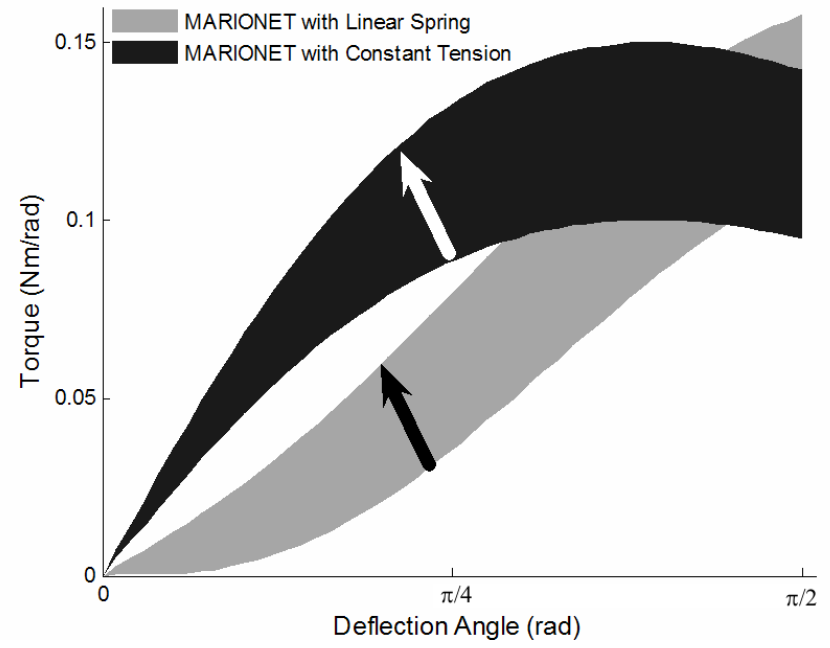

Fig. 4. Pretensioning acts as compliance control The MARIONET is capable of both equilibrium point and compliance control, all done mechanically. The Tensioner acts as a gain for compliance. Typical pretensioning values range from 0 to $5 \mathrm{~cm}$ for the $10 \mathrm{~N} / \mathrm{m}$ spring, and 1 to $1.5 \mathrm{~N}$ for cable tension.

version used steel cable with a servomotor maintaining constant tension, instead of an elastic cable. Tests found that the device behaves according to theory, producing accurate torque control and sufficient position control. Yet these experiments used an artificial arm and assumed a small amount of damping. How would the lack of damping in the physical device affect a real human user? Would the MARIONET be able to exert force fields on its subject?

We designed a brief pilot experiment to lead towards answering two questions: first, can the natural damping of the human elbow compensate for the lack of damping in the MARIONET, and second, can this device reliably exert both error enhancing and error reducing forces in a trajectory? For purposes of brevity and since too few subjects were used to draw any conclusions, we will only focus on the qualitative aspect of the first objective.

1) Methods: The six subjects in this experiment were asked to follow an 8-hump minimum-jerk velocity profile, varying in direction and speed. A position-controlled laser projected the desired trajectory onto an opaque white surface below (see Figure 5). The subject pool was divided evenly between three groups: Guidance, exerting correctional forces proportional to position error, Error Augmentation, amplifying position error by exerting proportional forces, and Control, where no forces are exerted at all. This ideal movement lasted 3.2 seconds and ranged between 0.5 to 2.3 radians. The force field in both cases was linear with a stiffness of $3 \mathrm{Nm} / \mathrm{rad}$. An LED attached to the handle provided visual feedback of the users hand position. A buzzer cued the beginning of a movement. Subjects rested whenever desired. Some of the reaches can be seen in Figure 6, where dark, thicker red lines indicate the desired trajectory, and the thinner, lighter blue lines are the individual trials.

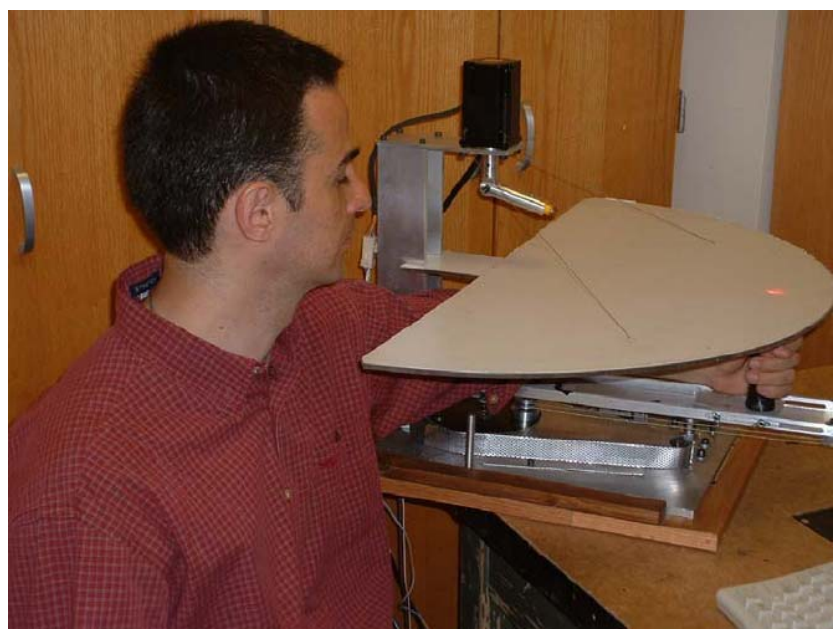

Fig. 5. Experimental setup with user and MARIONET proof-ofconcept.

2) Results and Conclusions: If the damping in the subjects' elbows was not enough to complement the MARIONET, then we would expect to see some oscillations in the Guidance group. If the MARIONET was too weak to affect the users' arms, we would not see a difference between any of the three groups. Figure 6 shows that neither situation is the case. From observation of the control group, note the shift in time between the desired and actual trajectories. This is expected since the user mimics the desired trajectory, instead of receiving haptic feedback. In the Guidance group, the errors in timing reduce significantly, exhibiting the device's ability to effectively exert guidance forces on its user. Due to relatively low bandwidth, the Guidance group has a small amount of error during quick positional changes. Finally, the Error Augmentation group looks different than the preceding two paradigms, actual trajectories being pushed away from desired trajectories. These results show that the device can move the human arm through a desired path without going unstable, and can exert both assistive and error enhancing forces.

\section{Design of Two-Joint MARIONET}

What has been established through previous work is the promise of a novel actuator. Translating this technology to a useful device cannot be accomplished without consulting on a consistent basis with the clinicians and patients on site. We plan on taking full advantage of the resources at the Rehabilitation Institute of Chicago during this design process. Design specifications are shown in Table I.

1) Design for Function: The two-joint MARIONET is a planar, upper extremity Manipulandum capable of use in various positions between the transverse (horizontal) and sagittal (vertical) planes. It is designed to fit a large variety of user dimensions, and be usable with the help of an untrained supervisor. The device is inherently stable and compliant, and also has online control of its endpoint stiffness and torque in both Cartesian and joint spaces. Hall effect position 


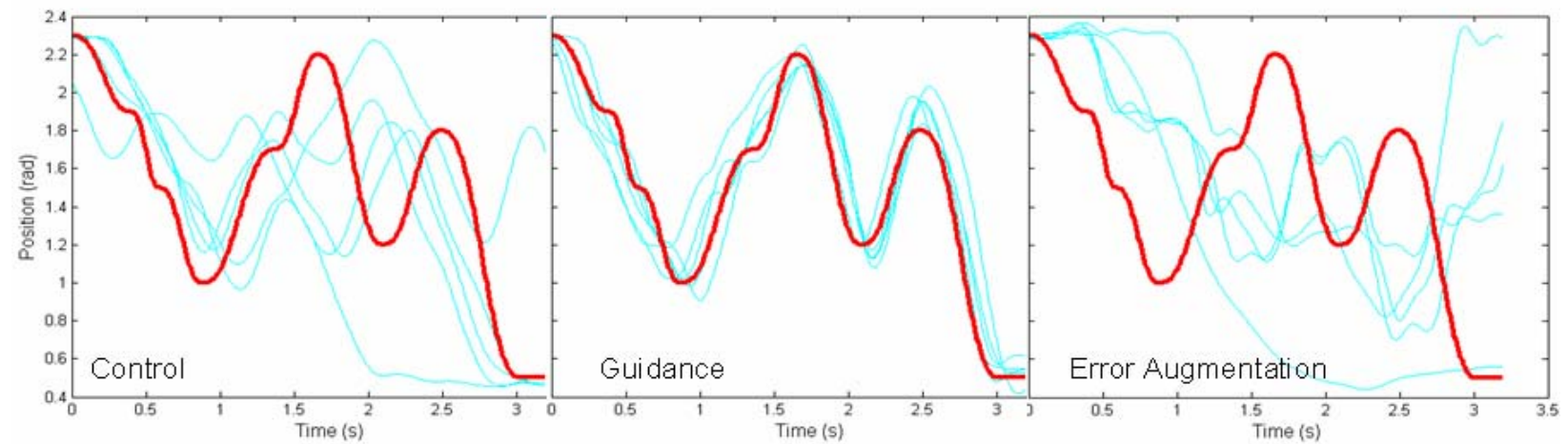

Fig. 6. Results of pilot experiment. Red lines show desired trajectory, and blue lines show the individual trials. Qualitatively, it seems that the MARIONET is capable of exerting a trajectory on its user even though it is a torque controlled device. Since the device itself has no damping, it depends on the damping of the user for stability.

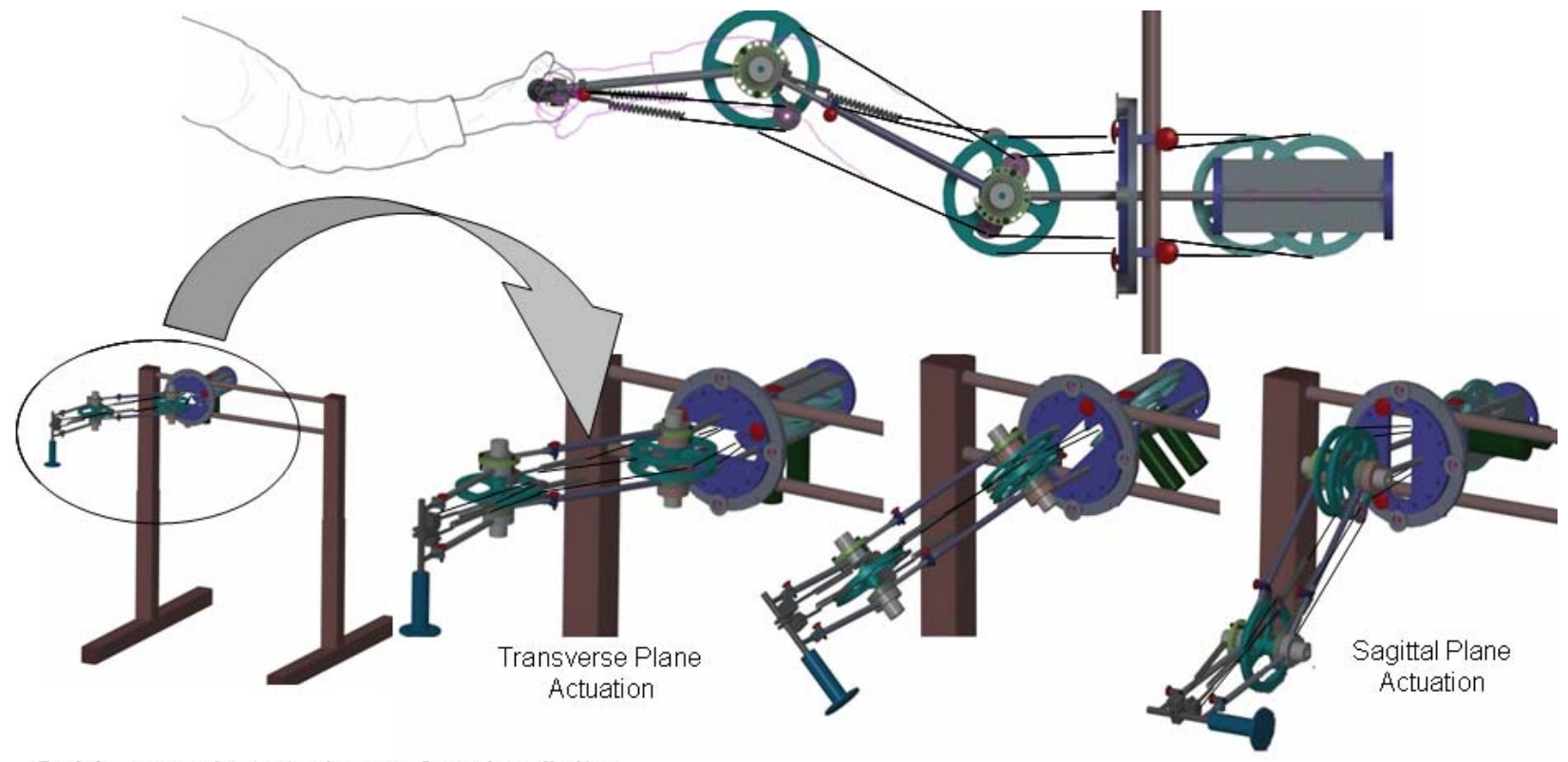

Cable guards not shown for simplicity

Fig. 7. Two-Joint MARIONET The top view of the device (above) shows two ways the human user can interface with the two-joint MARIONET. The user can adjust the linkages to colocate the joints with his/her own, or like the MIT-Manus, remain opposite the mechanism. The picture on the bottom shows that the MARIONET can be adjusted to operate in various planes between the sagittal and the transverse. It can also slide down a track to operate on either side of the body. The height of the device can be changed with telescoping struts. Small Tensioner motors located at the handle and at the distal joint are configured in series with a spring for online adjustable compliance. Adjustable mechanical stops (shown on each joint) and cable guards (not shown) are examples of some of the safety precautions taken.

sensors measure the position of each moving component, and as a result, are able to calculate velocity, acceleration, endpoint force and endpoint stiffness. Operation and data recording use a real time operating system. Since it is intended for home and clinical use, it is light and mobile, low maintenance, easy to use, safe, and relatively inexpensive. In terms of its proposed versatility of function, accessibility and cost, the MARIONET substantially exceeds other planar manipulanda, in large part due to cable actuation.

2) Designing With Cables: Although cable is lightweight, flexible, and capable of spanning multiple joints, there are many pitfalls that will result in a shorter lifespan. The design
TABLE I

DESIGN SPECIFICATIONS

\begin{tabular}{|c||c||c|}
\hline Design Criterion & Goal & Reason \\
\hline \hline Usable Workspace & $0.4 \mathrm{~m}^{2}$ & Human capability [14] \\
\hline Software & QNX RTOS & Simple, effective OS \\
\hline Weight & $<50 \mathrm{lbs}$. & Portability \\
\hline Cost & $<\$ 3000$ (parts) & Affordability \\
\hline Force Bandwidth & $8 \mathrm{~Hz}$ & Human capability \\
\hline Maximum force & $25 \mathrm{~N}$ & Max. experimental use \\
\hline Force Accuracy & $0.5 \mathrm{~N}$ & Just Noticeable Diff. [15] \\
\hline Position Accuracy & $5 \mathrm{~mm}$ & Just Noticeable Diff. [15] \\
\hline
\end{tabular}


of the MARIONET avoids practices that reduce the breaking strength of cable such as cable wrapping, small bending radii and angles, sharp increases in loading, contact with stationary objects, poor sheave (pulley) design, rope rotation (twisting), damage from cutting, kinking, or crushing, and various other design principles illustrated in handbooks and papers.

3) Design for Safety: The safety of the human user must come before any performance criteria. During the design (and redesign) of the MARIONET, several failure modes are addressed, such as cable failure, loss of control, electrical faults, etc. Safety measures such as cable guards, mechanical stops, emergency stop switches (both hardware and software) are examples incorporated into the design, anticipating some of the failure modes. As a check to this practice, we use a hazard assessment matrix defined by Fries [16], taking into account relative values for the occurrence, detectability and severity of the event. If the score reaches an unacceptable amount, the problem must be mitigated, or another path must be taken. After the device is built, intensive operation determines if the safety problems have been solved, and reveal any new problems to mitigate before proceeding to performance evaluation.

4) Design for Control, Ease of Operation and Low Maintenance: The MARIONET is controlled using four inputs: the position of the Rotator located at each of two joints, and the small Tensioner motors controlling the equilibrium position of the springs (see Figure 7). Since the mechanism is used in positions outside the transverse plane, it must have gravity compensation programmed into its operation. The Tensioners, adjusting endpoint stiffness, will be able to add more versatility in creating various force fields, and have control over position accuracy. Since the MARIONET will eventually be used at home, it must be small, lightweight, and easy to use by a healthy operator. Everyday adjustments like changing planes of operation will be accomplished nearly effortlessly by hand, and simple commands like turning the device on and getting it to start a basic algorithm must be easy as well. Finally, components most likely to fail such as cables, sensors, and motors are arranged in locations that make them easy to replace.

\section{FUTURE WORK}

This version of the MARIONET must pass through several stages before its intended use of widespread clinical and home rehabilitation. The first entails further design iterations with clinicians. Next will involve a performance examination, with tests such as torque accuracy and maximum exertion. We will test the device's ability to teach healthy subjects a new trajectory using guidance and error augmentation paradigms. We also plan to conduct studies on stroke patients using a similar experiment.

\section{ACKNOWLEDGMENTS}

The authors gratefully acknowledge the contribution of the American Heart Association and the NIH T32 HD 07418.

\section{REFERENCES}

[1] Volpe, B. et al. A novel approach to stroke rehabilitation; robot-aided sensorimotor stimulation. Neurology 54, 1938-1944 (2000).

[2] Krebs, H. I., Aisen, M. L., Volpe, B. T. \& Hogan, N. Quantization of continuous arm movements in humans with brain injury. Proceedings of the National Academy of Sciences of the United States of America 96, 4645-4649 (1999).

[3] Reinkensmeyer, D. J., Dewald, J. P. \& Rymer, W. Z. Guidance-based quantification of arm impairment following brain injury: a pilot study. IEEE Transactions on Rehabilitation Engineering 7, 1-11 (1999).

[4] Zinn M, Khatib O, Roth B, Salisbury JK A New Actuation Approach for Human Friendly Robot Design. International Journal of Robotics Research 5:113-122 (2003).

[5] Pratt GA, Williamson MM Series elastic actuators. IROS, p 399. (1995).

[6] Pratt J Virtual Model Control of a Biped Walking Robot. Dept. of Electrical and Computer Engineering. Cambridge: MIT. (1995).

[7] Paluska D Design of a Humanoid Biped for Walking Research. Cambridge: Massachusetts Institute of Technology. (2000).

[8] Yobotics! www.yobotics.com. (2005).

[9] Veneman JF, Ekkelenkamp R, Kruidhof R, Helm FCTvd, Kooij Hvd Design of a Series Elastic and Bowden cable-based actuation system for use as torque-actuator in exoskeleton-type training. International Conference on Rehabilitation Robotics (ICORR). Chicago, IL. (2005).

[10] Hollander KW, Sugar TG, Herring DE Adjustable Robotic Tendon using a Jack Spring. 9th International Conference on Rehabilitation Robotics. Chicago, IL. (2005).

[11] Hurst JW, Chestnutt JE, Rizzi AA. An Actuator with Physically Variable Stiffness for Highly Dynamic Legged Locomotion. International Conference on Robotics and Automation. New Orleans. (2004).

[12] Sulzer, J. S., Peshkin, M. A. \& Patton, J. L. Catastrophe and Stability Analysis of a Cable-Driven Joint. IEEE Engineering in Medicine and Biology Conference (EMBS). New York, NY, USA. (2006).

[13] Sulzer, J. S., Peshkin, M.A.\& Patton, J. L. MARIONET: An Exotendon-Driven Rotary Series Elastic Actuator for Exerting Joint Torque. IEEE-International Conference on Rehabilitation Robotics (ICORR) Chicago, IL, USA. (2005).

[14] Winter DA. Biomechanics and Motor Control of Human Movement, 2nd Edition. New York: John Wiley \& Sons. (1990).

[15] Brewer, B., Klatzky, R. \& Matsuoka, Y. Perceptual Limits for a Robotic Rehabilitation Environment Using Visual Feedback Distortion. IEEE Transactions on Neural Systems \& Rehabilitation Engineering (2005).

[16] Fries. Reliable Design of Medical Devices. Marcel Dekker, Inc. N.Y., (1997). 